\title{
The cardiac response to exercise in cirrhosis
}

\author{
F Wong, N Girgrah, J Graba, Y Allidina, P Liu, L Blendis
}

\begin{abstract}
Background-Impaired exercise capacity and oxygen consumption are common in cirrhosis.

Aim-To explore the relationship between possible myocardial dysfunction and exercise tolerance in cirrhosis.

Methods-Cardiac responses to exercise, using radionuclide angiography and graded upright cycle ergometry with oxygen consumption, were assessed before and after exercise in 39 cirrhotics patients and compared with 12 age and sex matched healthy volunteers. Baseline cardiac chamber dimensions and wall thickness, ejection fraction, and diastolic function were measured using two dimensional echocardiography is all subjects.

Results-Baseline diastolic dysfunction with prolonged isovolumic relaxation times $(p=0.02)$, left atrial enlargement, and left ventricular wall thickening were present in all cirrhotics $(p=0.02)$, despite increased mean ejection fraction. With graded exercise, cirrhotics achieved 71 (4)\% $(p=0.03)$ (pre-ascitics) and $46(3) \%$ $(\mathbf{p}<0.001)$ (ascitics) of predicted work loads, respectively, without significant increases in ejection fraction. The smaller absolute and percentage increases in cardiac output $(p=0.003)$ in the cirrhotics were associated with significantly reduced oxygen consumption $(\mathrm{p}=\mathbf{0 . 0 0 3})$ and anaerobic threshold $(\mathrm{p}<0.001)$, and correlated significantly with work and metabolic parameters.
\end{abstract}

Conclusions-Impaired exercise capacity in cirrhosis is associated with myocardial thickening and ventricular stiffness leading to decreased diastolic function, inotropic and chronotropic incompetence under conditions of stress, with metabolic consequences. This picture is compatible with the condition now known as cirrhotic cardiomyopathy.

(Gut 2001;49:268-275)

Keywords: cirrhosis; exercise tolerance; myocardial function; oxygen consumption

Cirrhosis has been known to be associated with a hyperdynamic circulation for the past 50 years, ${ }^{1}$ manifesting primarily as increased cardiac output and decreased systemic vascular resistance. ${ }^{1}$ More recently, cardiac structural and functional abnormalities have also been described in both pre-ascitic and ascitic cirrhotic patients of alcoholic and nonalcoholic aetiologies. ${ }^{23}$ These include hypertrophy of the myocardium, leading to a stiffer ventricle and hence diastolic dysfunction, ${ }^{2-4}$ and normal systolic function at rest but systolic incompetence under conditions of stress. ${ }^{5-7}$ This constellation of abnormalities has been termed "cirrhotic cardiomyopathy". ${ }^{3}$ This contrasts with alcoholic cardiomyopathy which is usually a dilated cardiomyopathy characterised by increased ventricular volumes and a decreased ejection fraction. ${ }^{8}$

The consequences of non-alcoholic cardiac abnormalities in cirrhosis have not been thoroughly investigated, and their clinical significance not yet defined. In the first of a series of studies, we showed that one aspect, decreased ventricular contractility, was exacerbated by $200 \mathrm{mmol}$ of sodium intake per day in some pre-ascitic cirrhotic patients. ${ }^{2}$ Impaired exercise capacity and oxygen consumption are common in cirrhosis, even in the absence of overt cardiopulmonary disease. We propose that the presence of specific cirrhotic cardiac abnormalities results in a reduced cardiac response to exercise, which in turn leads to decreased oxygen delivery with metabolic consequences. Therefore, this study seeks to explore the relationship between cirrhotic cardiac abnormalities and exercise performance in cirrhosis.

\section{Materials and methods}

Ethics approval for the study was granted by the ethics committee of the Toronto General Hospital, University Health Network. All control subjects and cirrhotic patients gave written informed consent for the study.

\section{PATIENTS}

Thirty nine patients ( 37 males, two females) with biopsy proven cirrhosis were recruited from the general hepatology and pre-transplant clinics of the Toronto General Hospital. Eighteen patients had no history of ascites or diuretic use. Absence of ascites was confirmed by ultrasound before enrollment. These patients were therefore termed pre-ascitic cirrhotic patients. The remaining 21 patients had obvious ascites clinically and this was confirmed on ultrasound. Ten pre-ascitic and 14 ascitic patients had alcoholic cirrhosis but had abstained from alcohol for at least six months prior to enrollment. This was documented by questionnaires to the patients and their families during repeat clinic visits and persistently near

Abbreviations used in this paper: $\mathrm{E} / \mathrm{A}$ ratio, (E velocity=early maximal ventricular filling velocity, A velocity=late diastolic or atrial velocity); $\mathrm{EF}$, ejection fraction; ECG, electrocardiograph; FEF25-75, forced expiratory flow from $25-75 \%$ of vital capacity; $\mathrm{FEV}_{1}$ forced expiratory volume in one second; FVC, forced vital capacity; $\mathrm{VO}_{2}$, oxygen consumption; $\mathrm{VO}_{2} \max$, oxygen consumption at maximal exertion; RNA, radionuclide angiography; AT, ventilatory anaebolic threshold. 
Table 1 Baseline demographics of control subjects and cirrhotic patients

\begin{tabular}{llll}
\hline & Controls & Pre-ascitics & Ascites \\
\hline $\mathrm{n}$ & 12 & 18 & 21 \\
Age $(\mathrm{y})$ & $49(3)$ & $50(2)$ & $52(3)$ \\
Sex $(\mathrm{M} / \mathrm{F})$ & $10 / 2$ & $18 / 0$ & $19 / 2$ \\
Haemoglobin $(\mathrm{g} / \mathrm{l})$ & $139(4)$ & $134(3)$ & $120(4)$ \\
Haematocrit & $0.41(0.01)$ & $0.39(0.01)$ & $0.35(0.01)$ \\
INR & $1.04(0.02)$ & $1.41(0.06)^{\star}$ & $1.69(0.09)^{\star \star}$ \\
Albumin $(\mathrm{g} / \mathrm{l})$ & $45(1)$ & $38(1)$ & $31(2)^{\star}$ \\
Bilirubin $(\mu \mathrm{mmol} / \mathrm{l})$ & $11(2)$ & $29(3)$ & $42(6)^{\star \star}$ \\
BMI (kg/m $\left.{ }^{2}\right)$ & $26.3(0.9)$ & $26.5(0.4)$ & $26.1(0.7)$ \\
Child-Pugh score & - & $5.8(0.2)$ & $10.1(0.5) \dagger$ \\
Aetiology of cirrhosis & & & \\
$\quad$ Alcohol & & 7 & 14 \\
$\quad$ HCV & & - & 2 \\
$\quad$ HBV & & - & 1 \\
$\quad$ Cryptogenic & & - & 1 \\
$\quad$ Drug induced & & & 1 \\
$\quad$ Autoimmune hepatitis & & &
\end{tabular}

INR, international normalised ratio; BMI, body mass index; HCV, hepatitis C virus; HBV, hepatitis $\mathrm{B}$ virus.

${ }^{\star} \mathrm{p}<0.05,{ }^{\star \star} \mathrm{p}<0.01$ versus controls; $\mathrm{tp}<0.05$ ascitic versus pre-ascitic cirrhotic patients.

normal serum gamma glutamyl transferase levels. Cirrhosis was related to hepatitis C infection in nine patients and hepatitis B infection in another two. Two patients had cryptogenic cirrhosis while the remaining two patients had autoimmune hepatitis and drug induced cirrhosis as a result of flutamide use, respectively. All were ambulatory patients, defined as giving a history of taking frequent exercise such as walking more than $1-2 \mathrm{~km}$ regularly. Patients with gross ascites, which prevented the use of an upright cycle ergometry, were excluded. Similarly, patients with tense ascites, which required regular paracenteses at intervals of two weeks or less, were excluded. All patients were stable and free of gastrointestinal bleeding within the previous three months. A negative hypertensive, cardiac, and pulmonary history, normal examination by a cardiologist, normal electrocardiograph (ECG), chest $x$ ray, spirometry, and oximetry were mandatory for inclusion in the study. The following criteria were used to exclude the presence of pulmonary diseases: forced expiratory volume in one second $\left(\mathrm{FEV}_{1}\right) \geqslant 80 \%$ of predicted, forced vital capacity (FVC) $\geqslant 80 \%$ of predicted, forced expiratory flow from $25 \%$ to $75 \%$ of vital capacity (FEF25-75) $\geqslant 70 \%$ of predicted, and oxygen saturation $\geqslant 95 \%$ on oximetry. ${ }^{9}$ Patients whose body mass index exceeded 2 SDs of normal $\left(>27.8 \mathrm{~kg} / \mathrm{m}^{2}\right.$ for men, $>27.3 \mathrm{~kg} / \mathrm{m}^{2}$ for women) were excluded. Likewise, patients who were cachectic because of their liver disease (whose body mass index was below 2 SDs of normal) or who were significantly anaemic, as defined as a haemoglobin concentration less than $110 \mathrm{~g} / \mathrm{l}$, were also excluded. A haematocrit of $>30 \%$ was required for enrollment. Tobacco use, apart from in the remote past, was also an exclusion criterion.

Twelve age and sex matched healthy nonsmoking individuals with no history of cardiac or pulmonary disease together with a normal chest $x$ ray, ECG, and blood pressure served as controls. In particular, the possibility of other causes of heart disease were excluded. Therefore, neither patients nor controls gave a history suggestive of cardiac ischaemia, hypertension, or had evidence of valvular heart disease, haemochromatosis, or amyloidosis.
Invasive investigations such as coronary angiography, to exclude coronary vessel disease, or myocardial biopsy, to exclude iron overload or amyloidosis, were not performed because of ethical considerations. Control subjects had to fulfill the same body mass index criteria and abstain from alcohol for one month prior to the study. To avoid the confounding problem of the control subjects being relatively less "detrained" than the cirrhotic patients, the control subjects were recruited from the Section of the Chronically Unemployed, Department of Welfare and Social Security. Individuals from this section suffer from chronic inactivity. Control subjects were specifically questioned about their daily activities, and were matched for the same amount of daily walking and exercise. Demographics of all study subjects and baseline parameters, including Child-Pugh score in all cirrhotic patients, are given in table 1 .

\section{STUDY DESIGN}

The study was performed with the control subjects and pre-ascitic cirrhotic patients consuming a metabolic diet of $200 \mathrm{mmol}$ of sodium/1.5 litre of fluid per day for seven days. This sodium intake was chosen because subtle cardiac dysfunction in some pre-ascitic cirrhotic patients has been shown to be more obvious as a result of the volume expansion associated with dietary sodium challenge. ${ }^{2}$ Furthermore, both controls and pre-ascitic cirrhotics have been shown to achieve a steady state after consuming this diet for seven days ${ }^{10}$ : this would ensure that a change in volume status was not introduced as a confounding factor in these two groups. In contrast, ascitic cirrhotic patients avidly retain sodium even on a low sodium intake; they were therefore placed on $20 \mathrm{mmol}$ of sodium/1.0 litre of fluid per day diet for seven days prior to the study. Day 1 of the study was the first day of the diet. All medications that could potentially affect cardiac function or volume status such as $\beta$ blockers or diuretics were withheld from day 1 . None of the ascitic patients received a paracentesis for two weeks prior to the study.

All study subjects underwent (i) two dimensional echocardiograph to assess cardiac structure and diastolic function; (ii) radionuclide angiography (RNA), in which the patient's own red blood cells are labelled with radioactive technetium and reinfused, so that the chest can be scanned to assess non-invasively vascular volumes of the various chest compartments including cardiac chamber volumes and ejection fraction (EF), and (iii) an upright cycle ergometry with repeat RNA measurements to assess the cardiac response to exercise with simultaneous oxygen consumption $\left(\mathrm{VO}_{2}\right)$ calculations.

PROTOCOL

All subjects were monitored with daily weights from day 1 to day 7 to ensure that a steady state of weight and hence volume status had been reached for at least three days prior to the study. Twenty four hour urine collections to determine urinary sodium excretion were performed on days 5,6 , and 7 as confirmation of 
attainment of this steady state. The study was then performed on days 8 and 9 of each study group's respective diet.

On day 8 of the study, all subjects underwent a transthoracic two dimensional echocardiograph examination performed by the same echocardiographer (JG). Measurements of left ventricular systolic and diastolic chamber dimensions, interventricular septal thickness, and left ventricular relative wall thickness (the thickness of the posterior wall plus the thickness of the interventricular septum relative to the internal diameter of the ventricle) were made as assessments of cardiac structure. ${ }^{11}$ Diastolic function was assessed by measuring the $\mathrm{E} / \mathrm{A}$ ratio ( $\mathrm{E}$ velocity=early maximal ventricular filling velocity, A velocity=late diastolic or atrial velocity), a reflection of the degree of impairment of diastolic relaxation; isovolumic relaxation time (the period of time from closure of the aortic valve to opening of the mitral valve); and deceleration time (the time period during which the ventricle inflow decelerates to a complete stop). An increase in isovolumic ventricular relaxation time and deceleration time, and a decreased $\mathrm{E} / \mathrm{A}$ ratio suggest impedance to left ventricular filling ${ }^{3}$ or increased ventricular stiffness.

On day 9 of the study, three hours after their usual breakfast, all subjects underwent an upright RNA study for measurements of cardiac chamber volumes and EF, a measure of systolic function. The best septal view was used to determine the end systolic and end diastolic volumes using the modified Links method. ${ }^{12}$ These were used for calculation of stroke volume and hence cardiac output. This was followed by a graded exercise test using upright cycle ergometry to determine the maximal workload. RNA measurements were repeated to allow calculation of the cardiac response to exercise at a workload corresponding to $70 \%$ of maximal exercise as determined in the graded exercise test. This workload was maintained until a plateau in oxygen consumption was obtained to ensure a steady state measurement. ECG, blood pressure, and oximetry were monitored continuously throughout the study. Breath by breath analysis of oxygen uptake and carbon dioxide release were determined using a metabolic cart to allow calculations of $\mathrm{VO}_{2}$ and anaerobic threshold (AT), an objective indicator of cardiovascular oxygen delivery and fitness. All exercise tests were supervised by either FW or NG and performed by YA.

\section{PROCEDURES}

(i) Two dimensional echocardiography

A complete transthoracic echocardiographic examination was performed using commercially available cardiac ultrasound machines (Hewlett Packard, Andover, Massachusetts, USA). Patients were placed in the left lateral decubitus position and standard parasternal, apical, and substernal views were obtained. Pulsed and colour flow Doppler was used to interrogate mitral and pulmonary venous inflows, and isovolumic relaxation and deceleration times. All images were then recorded to magnetic-optical discs and VHS tapes for offline analysis.

(ii) Radionuclide angiography

Detailed description of the technique of cardiac chamber volume measurements using RNA can be found elsewhere. ${ }^{13}$ Briefly, red blood cells were labelled using Tc-99m pertechnetate. Cardiac volumes were measured based on regional activity corrected for attenuation. EF and cardiac volumes were analysed using semiautomated software. Quality assurance studies in our nuclear cardiology laboratory have established the standard error of the estimate of left ventricular ejection fraction calculation to be less than $2 \%$ using the semiautomated technique. The standard error of the estimate of ventricular volume calculation is less than $5 \mathrm{ml}^{14}$

(iii) Bicycle cardiopulmonary exercise test The patient was seated upright on a Quinton automated exercise cycle, interfaced with Q5000 or equivalent electronic control system (Quinton Instrument Company, Seattle, Washington, USA). A tight fitting mouthpiece with a sealed nasal passage was then placed on the patient. Expired gases were collected and measured on a breath by breath basis on a Sensormedics metabolic cart (Sensormedics Metabolic Carts, Yorba Linda, California, USA). The gas analyser was first calibrated against standard cylinders of reference gases. After a one minute warm up of pedalling against a free load, the patient then pedalled against a continuously increasing exercise load. Continuous increments equivalent to 50 kilopon metre $(\mathrm{kpm}) / 30$ seconds was applied until symptom exhaustion. Breath by breath measurements of expired gases provided data for the determination of $\mathrm{VO}_{2}$ max (oxygen consumption at maximal exertion) and the ventilatory AT (the point at which lactic acid is produced by the body) using the gas exchange threshold (V slope) method. ${ }^{15}$

STATISTICAL ANALYSIS

All results are expressed as mean (SEM). For each parameter, differences between the three study groups were assessed by analysis of variance. Neuman-Keul's multiple range testing was used to analyse the differences between the alcoholic and non-alcoholic patients. Regression analysis was used to determine the relationship between the cardiac parameters and work parameters. A $\mathrm{p}$ value $<0.05$ was considered statistically significant.

\section{Results}

During the study period of July 1998 to January 2000, 102 consecutive cirrhotic patients with or without ascites were assessed for their suitability for exercise testing with baseline standard resting pulmonary function. Thirty nine serial patients were identified and therefore enrolled into the study. Their pulmonary function results are reported in table 2. All controls and cirrhotic patients also had normal baseline ECG, chest $x$ ray, and oximetry. 
Table 2 Baseline standard resting pulmonary function of control subjects and cirrhotic patients

\begin{tabular}{lccc}
\hline & Controls & Pre-ascitics & Ascitics \\
\hline $\mathrm{n}$ & 12 & 18 & 21 \\
FEV $_{1}$ (\% of predicted) & $94(8)$ & $93(4)$ & $89(4)$ \\
FVC (\% of predicted) & $101(7)$ & $96(3)$ & $84(5)$ \\
FEV $_{1} /$ FVC & $0.75(0.03)$ & $0.77(0.02)$ & $0.79(0.01)$ \\
FEF25-75 (\% of predicted) & $82(9)$ & $84(7)$ & $74(5)$ \\
Oxygen saturation & $98.2(0.4)$ & $96.6(0.4)$ & $96.4(0.6)$
\end{tabular}

$\overline{\mathrm{FEV}_{1} \text {, forced expiratory volume in one second; FVC, forced vital capacity; FEF25-75, forced }}$ expiratory flow from 25 to $75 \%$ capacity.

Table 3 Baseline haemodynamics, cardiac structure, and cardiac function of control subjects and cirrhotic patients

\begin{tabular}{lccc}
\hline & Controls & Pre-ascitics & Ascitics \\
\hline $\mathrm{n}$ & 12 & 18 & 21 \\
Heart rate (beats/min) & $65(4)$ & $71(2)$ & $73(4)$ \\
Mean arterial blood pressure (mm Hg) & $92(4)$ & $90(2)$ & $87(2)$ \\
Cardiac index (1/min/m ${ }^{2}$ ) & $2.33(0.22)$ & $2.19(0.17)$ & $2.40(0.20)$ \\
Systemic vascular resistance (dyn s/cm $\left.{ }^{5}\right)$ & $1962(166)$ & $1866(163)$ & $1648(203)$ \\
Ejection fraction (\%) & $59.7(2.1)$ & $64.7(2.6)^{\star}$ & $65.9(2.0)^{\star}$ \\
End diastolic volume (ml) & $103(12)$ & $99(7)$ & $82(6)^{\star}$ \\
End systolic volume (ml) & $46(6)$ & $34(4)$ & $26(3)^{\star \star}$ \\
Isovolumic relaxation time (ms) & $78.4(3.7)$ & $89.9(2.8)^{\star}$ & $94.6(4.0)^{\star \star}$ \\
Deceleration time (ms) & $218(11)$ & $208(12)$ & $236(18)$ \\
E/A ratio & $1.34(0.11)$ & $1.20(0.10)$ & $0.97(0.05)^{\star}$ \\
Left atrial size (mm) & $36.3(6.2)$ & $42.7(1.3)^{\star}$ & $41.6(0.9)^{\star}$ \\
Interventricular septal thickness (mm) & $8.8(0.3)$ & $10.6(0.3)^{\star}$ & $10.7(0.4)^{\star}$ \\
Left ventricular relative wall thickness & $0.38(0.01)$ & $0.42(0.01)$ & $0.46(0.02)^{\star \star}$ \\
\hline
\end{tabular}

${ }^{\star} \mathrm{p}<0.05,{ }^{\star \star} \mathrm{p}<0.01$ versus controls; $\mathrm{tp}<0.05$ ascitic versus pre-ascitic cirrhotic patients.

BASELINE CARDIOVASCULAR ASSESSMENT

Haemodynamics

Both controls and cirrhotics had similar baseline systemic haemodynamics, with ascitic cirrhotic patients tending towards a more hyperdynamic circulation with a higher resting heart rate, lower mean arterial pressure, higher cardiac output, and lower systemic vascular resistance (table 3 ).

Systolic and diastolic function

No wall motion abnormalities or valvular lesions were noted during the echocardiographic examination in controls or cirrhotics. $\mathrm{EF}$ was in the normal range in all three study groups, with values in cirrhotic patients being significantly higher than those in controls (table 3). This was associated with significantly smaller left ventricular dimensions in ascitics, both at the end of diastole and at the end of systole (table 3 ).

Isovolumic relaxation time was significantly prolonged in both pre-ascitic and ascitic patients while deceleration time showed a trend towards an increase in ascitic cirrhotic patients (table 3). Left atrial diameter was significantly increased in both pre-ascitic and ascitic patients compared with controls (table 3). E/A ratio, a measure of the degree of impairment of diastolic relaxation, was only significantly reduced in ascitic patients $(\mathrm{p}<0.05 v$ controls $)$ (table 3).

\section{Cardiac structure}

Interventricular septal thickness was significantly increased in both pre-ascitic and ascitic cirrhotic patients compared with controls (table 3). Left ventricular relative wall thickness (left ventricular wall thickness plus septal thickness relative to the internal dimensions of the left ventricle) was also significantly greater
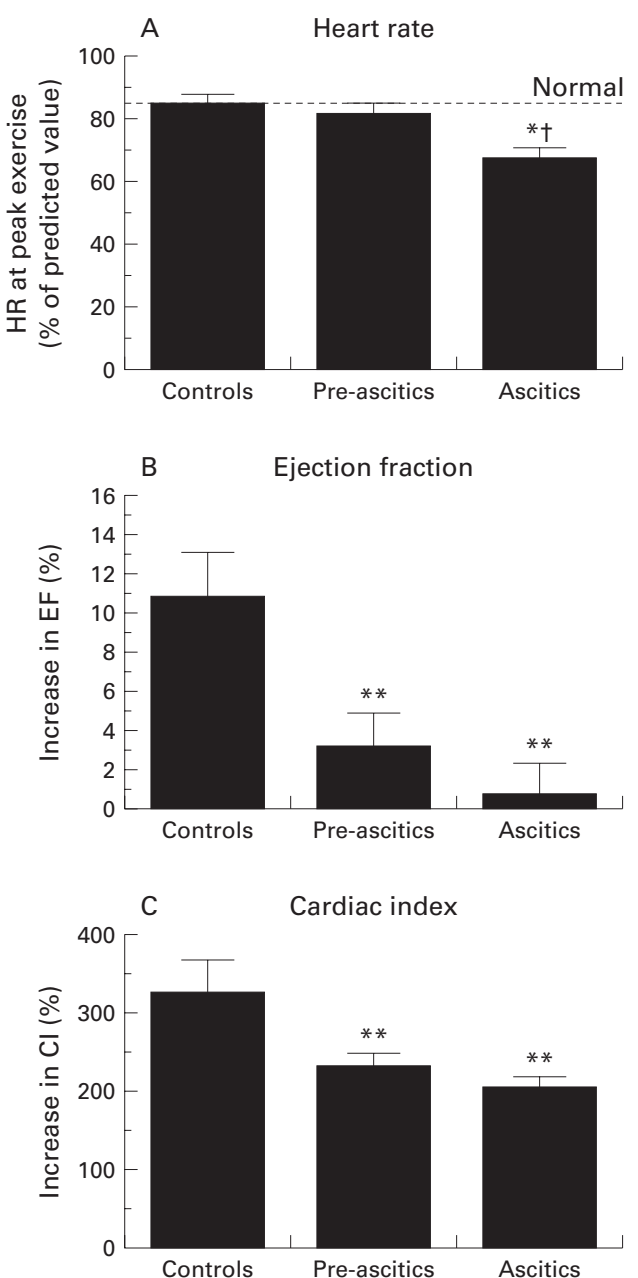

Figure 1 Increase in $(A)$ heart rate $(H R),(B)$ ejection fraction (EF), and $(C)$ cardiac index (CI) with peak exercise in healthy controls, pre-ascitic, and ascitic cirrhotic patients. HR is expressed as a percentage of predicted values. Values are mean (SEM). ${ }^{\star} p<0.05,{ }^{\star \star}{ }^{*}<0.01$ compared with controls; $t p<0.05$ pre-ascitics compared with ascitic cirrhotic patients.

in ascitic patients only compared with controls (table 3).

WORK PERFORMANCE

With symptom limited exercise, cirrhotic patients exercised for shorter periods and achieved less peak oxygen consumption and workload compared with controls. The differences were statistically significant in ascitic cirrhotic patients. For example, control subjects performed 932 (67) kpm of work for $8.3(0.8)$ minutes before they gave up because of exhaustion. Pre-ascitic cirrhotic patients worked for $7.3(0.6)$ minutes and achieved 834 (49) $\mathrm{kpm}$ while ascitic cirrhotic patients worked for $3.9(0.3)$ minutes $(\mathrm{p}<0.001 v$ controls and pre-ascitic cirrhotics) and achieved 493 (27) kpm of work ( $\mathrm{p}<0.001 v$ controls and pre-ascitic cirrhotics). When the results were analysed using the percentage of predicted work based on age and sex, controls achieved 88 (5)\% of what was predicted whereas pre-ascitic cirrhotic patients achieved 71 (4)\% of their predicted value ( $\mathrm{p}=0.03 v$ controls), and ascitic cirrhotic patients achieved 46 (3)\% of their predicted value $(\mathrm{p}<0.001 v$ controls 

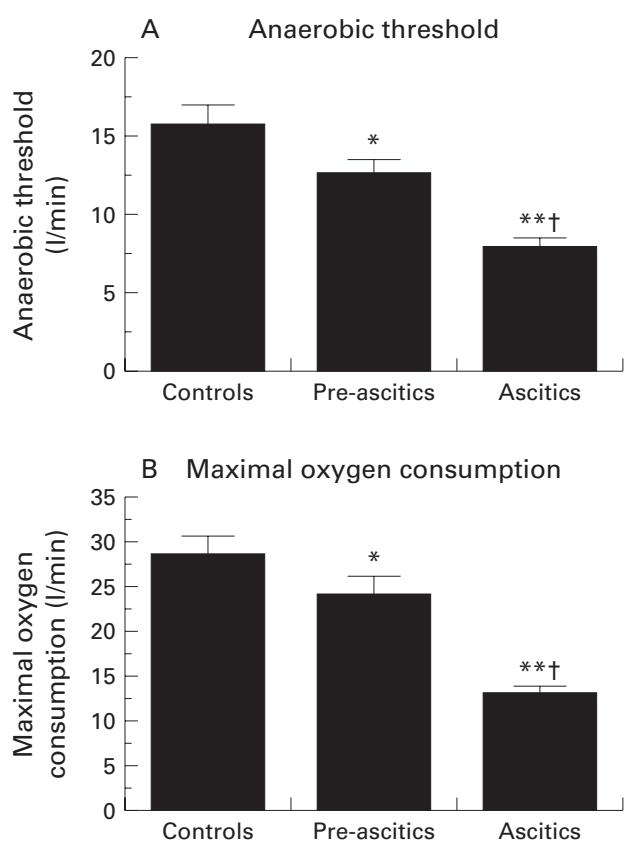

Figure 2 (A) Anaerobic threshold and (B) oxygen consumption with exercise in healthy controls, pre-ascitic, and ascitic cirrhotic patients. Values are mean (SEM). ${ }^{\star} p<0.05,{ }^{\star} x p<0.01$ compared with controls; $t p<0.05$ pre-ascitics compared with ascitic cirrhotic patients.

and pre-ascitic cirrhotics). More than $80 \%$ of patients in all three groups stopped exercising due to leg fatigue.

\section{CARDIAC AND METABOLIC RESPONSES TO}

EXERCISE

Cirrhotic patients demonstrated a significantly reduced cardiac response to exercise (fig 1A). For example, peak heart rate was 147 (6) beats $/ \mathrm{min}$ in controls, 136 (6) beats $/ \mathrm{min}$ in pre-ascitics, and 110 (4) beats/min in ascitics $(\mathrm{p}<0.05 v$ controls and pre-ascitics). When the increase in EF with exercise in cirrhotics was compared with controls, this was significantly reduced (fig 1B). Consequently, both the absolute and percentage increases in cardiac output in cirrhotic patients were also significantly decreased compared with controls (cardiac index at peak exercise was $7.43(0.79) 1 / \mathrm{min} / \mathrm{m}^{2}$ in controls, $4.81(0.44) \mathrm{l} / \mathrm{min} / \mathrm{m}^{2}$ in pre-ascitics $\left(\mathrm{p}<0.05 v\right.$ controls), and $4.30(0.26) 1 / \mathrm{min} / \mathrm{m}^{2}$ in ascitics $(\mathrm{p}<0.05 v$ controls)) (fig $1 \mathrm{C})$.

When the cardiac response of patients with an alcoholic aetiology of their cirrhosis was compared with that of patients with nonalcoholic cirrhosis there was no difference between the two subsets of patients (table 4).
Maximal $\mathrm{VO}_{2}$, expressed either as an absolute value or as a percentage of predicted values, was abnormal in cirrhotics, with percentage predicted values being significantly decreased in both pre-ascitic and ascitic patients compared with controls (fig $2 \mathrm{~B}$ ), and that of ascitic patients being significantly worse than that in pre-ascitic patients. AT was significantly reduced in cirrhotic patients compared with controls (fig 2A). Ascitic cirrhotic patients were again significantly worse than pre-ascitic cirrhotic patients.

There was a significant correlation between $\mathrm{E} / \mathrm{A}$ ratio and anaerobic threshold $(r=0.41$, $\mathrm{p}<0.01)$ and maximal oxygen consumption $(r=0.64, \mathrm{p}<0.01)$ in cirrhotic patients.

\section{Discussion}

In this study, we have demonstrated the presence of subtle cardiac abnormalities in a selected group of cirrhotic patients with or without ascites, irrespective of the aetiology of cirrhosis. These abnormalities were characterised by the presence of myocardial thickening, atrial enlargement, diastolic dysfunction, with or without diminution of left ventricular dimension, in the presence of preserved baseline systolic function. Furthermore, cirrhotic patients demonstrated a significant reduction in exercise capacity and abnormal oxygen consumption. They also had an early ventilatory anaerobic threshold which was reached in all cases, and which correlated with diastolic dysfunction.

There may be several explanations for the reduced exercise capacity in cirrhosis. Inactivity, bed rest, obesity, or cachexia could reduce the patient's ability to exercise fully. This was overcome by selecting only ambulatory patients who were within a limited range of body mass index and self caring at home. Control subjects were specifically matched for body mass, age, sex, and the degree of usual daily activities to eliminate a training or detraining effect. ${ }^{1617}$ Care was taken to select patients without pulmonary hypertension and with normal pulmonary function, as measured by spirometry and normal oxygen saturation on room air, thereby eliminating abnormal pulmonary function as a cause of the reduced cardiopulmonary response to exercise. Anaemia can decrease oxygen carrying capacity thereby reducing exercise capacity. ${ }^{18}$ The haematocrit in pre-ascitic cirrhotic patients was the same as in controls, despite the presence of reduced exercise capacity. Although the haematocrit

Table 4 Cardiac response to exercise in alcoholic and non-alcoholic cirrhotic patients

\begin{tabular}{|c|c|c|c|c|}
\hline & \multicolumn{2}{|l|}{ Pre-ascitics } & \multicolumn{2}{|l|}{ Ascitics } \\
\hline & Alcoholic & Non-alcoholic & Alcoholic & Non-alcoholic \\
\hline $\mathrm{n}$ & 10 & 8 & 14 & 7 \\
\hline Exercise time (min) & $6.73(0.78)$ & $8.47(0.63)$ & $3.96(0.30)$ & $4.30(0.78)$ \\
\hline Maximal work performed (kilopon meter) & $795(65)$ & $933(62)$ & $479(25)$ & $521(65)$ \\
\hline Heart rate at peak exercise ( $\%$ of predicted) & $78(4)$ & $84(5)$ & $70(3)$ & $64(4)$ \\
\hline Increase in $\mathrm{EF}$ with exercise $(\%)$ & $3.1(0.6)$ & $2.5(0.9)$ & $2.2(1.4)$ & $2.3(1.5)$ \\
\hline Increase in CI with exercise (\%) & $246(24)$ & $221(20)$ & $212(19)$ & $225(22)$ \\
\hline Anaerobic threshold $(1 / \mathrm{min})$ & $12.0(1.0)$ & $13.8(1.3)$ & $8.3(0.8)$ & $8.4(0.7)$ \\
\hline Oxygen consumption $(1 / \mathrm{min} / \mathrm{kg})$ & $23(2)$ & $28(3)$ & $14(1)$ & $13(2)$ \\
\hline
\end{tabular}

EF, ejection fraction; CI, cardiac index. 
tended to be lower in ascitic patients, the difference was not significant.

The most noticeable cardiac abnormality was diastolic dysfunction. This was manifested by an increased isovolumic relaxation time and reduced E/A ratio and a dilated left atrium, most probably from increased impedance to venous inflow. Although all parameters of diastolic function measured were still within "normal limits", they were significantly different from controls, again emphasising the subtle nature of the condition. The aetiology of diastolic dysfunction could be multifactorial. Myocardial hypertrophy is well documented in cirrhosis. ${ }^{1920}$ This could be due to the presence of increased levels of various growth factors, such as angiotensin II, ${ }^{21} 22$ endothelin, ${ }^{23}$ or cytokines such as tumour necrosis factor, ${ }^{24}$ or increased sympathetic output with $\alpha$ stimulation of the myocyte. ${ }^{25}$ The hypertrophied and therefore stiff myocardium not only impedes ventricular filling but also impairs calcium removal, with consequent depressed active relaxation. ${ }^{26}$ Coupled with myocyte abnormalities, there could also be increased interstitial fibrosis ${ }^{27}$ which further contributes to diastolic dysfunction.

Diastolic dysfunction in these patients was associated with an abnormal inotropic and chronotropic response to exercise despite normal resting systolic function. EF remained unchanged, contrary to that predicted by Starling's law, which states that greater venous return with exercise with resultant stretching of the myocardium should result in an increase in EF rather than minimal or no change, although $\mathrm{EF}$ in cirrhotics, in addition to intrinsic contractility, reflects the potentially altered preload and afterload conditions within which the heart functions. However, the lower afterload due to systemic arterial vasodilatation and the higher preload due to increased venous return in cirrhotic patients should have yielded a much higher EF. The fact that cirrhotics, despite their loading abnormalities in favour of a higher EF, did not have a higher resting EF or an appropriate increase in EF during exercise raises the possibility of an inappropriate contractile response and possible impairment. Similarly, loading conditions in cirrhosis can also affect diastolic filling parameters. However, again, in the setting of higher preload and lower afterload, diastolic filling should be enhanced rather than impaired. Therefore, the observation of impaired diastolic filling is consistent with a possible intrinsic abnormality of the ventricle despite favourable loading conditions.

Submaximal increases in cardiac output following exercise were also observed. One could argue that in ascitic cirrhotic patients the presence of ascites interferes with the patient's ability to exercise and therefore the cardiac response would be reduced. However, similar reductions in cardiac response were observed in both pre-ascitic and ascitic cirrhotic patients. Suboptimal cardiac response following exercise in both alcoholic and non-alcoholic cirrhotic patients compared with controls has previously been reported. ${ }^{7}$ Laffi et al, using active tilt to assess the isotropic and chronotropic response in a group of non-alcoholic cirrhotic patients, also reported a significantly decreased stroke volume with tilt, leading to a reduced cardiac index despite a significant increase in heart rate. ${ }^{6}$ The inotropic and chronotropic dysfunction in cirrhosis appears to be related to abnormalities in the sympathetic activation of the myocardium. The sympathetic signalling cascade is abnormal in cirrhosis, as demonstrated by Ma et al and Lee et al, who found a reduction in $\beta_{1}$ adrenoreceptor density in the plasma membrane of myocytes, ${ }^{28}{ }^{29}$ associated with a decrease in $\beta$ adrenoreceptor stimulated cAMP production. $^{30} 31$

Maximal oxygen consumption correlates with oxygen delivery and is dependent on cardiac output and peripheral oxygen utilisation. An early anaerobic threshold with relatively unchanged pulmonary function tests is compatible with a cardiovascular factor being involved in the limitation to exercise. This could be due to peripheral vascular abnormalities or cardiac diastolic dysfunction. In the normal response to exercise, there is an increase in cardiac volume to allow the appropriate increase in left ventricular stroke volume. However, the presence of a smaller cardiac volume due to diastolic dysfunction, such as seen in this study, would lead to significant smaller increases in stroke volume with exercise.

Circulatory changes, such as those found in the pulmonary circulation, could also contribute to the reduced anaerobic threshold in cirrhotic patients. In normal individuals, the elevated oxygen flux during exercise is the result of an increase in cardiac output, with a consequent increase in pulmonary blood flow due to recruitment of the segments of capillary bed not perfused at rest. Most of the increased oxygen flux is directed to the muscles, secondary to a marked fall in vascular resistance in the vascular bed of active muscles. Generalised sympathetic activation causes vasoconstriction in other non-active vascular beds. ${ }^{32}$ Pulmonary vasodilatation with pulmonary $\mathrm{A}-\mathrm{V}$ shunts is well documented in cirrhosis. ${ }^{33-35}$ In contrast with controls, ${ }^{32}$ Agusti et al demonstrated no change in the pulmonary circulation in cirrhosis with exercise, with no further increase in pulmonary blood flow or fall in pulmonary vascular resistance. ${ }^{36}$ They attributed this to the presence of an almost completely dilated and recruited resting pulmonary vascular bed at rest and therefore any further increase in cardiac output most likely would result in shunting of blood to other vascular beds. To date, there has been no report on the response of the skeletal muscle vascular bed to exercise in cirrhosis. Increase in muscle blood flow during dynamic exercise is nitric oxide mediated. ${ }^{37}{ }^{38}$ Nitric oxide production is enhanced in cirrhosis at rest. ${ }^{39}{ }^{40}$ This in turn may result in decreased myocardial function. ${ }^{41}{ }^{42}$ Similarly, an already vasodilated skeletal muscle vascular bed may only undergo limited further dilatation with exercise. Finally, hyporesponsiveness 
to sympathetic stimulation, exacerbating vasodilatation, is well documented in some vascular beds in cirrhosis, including the renal circulation. ${ }^{43}$

It is possible that alcoholic cardiomyopathy could mimic some of the findings in this study. ${ }^{44}$ However, in agreement with previous reports, similar abnormal cardiac responses to exercise were observed in both abstinent alcoholic and non-alcoholic cirrhotic patients. ${ }^{7}$ Furthermore, the findings of this study support previous reports of alcoholic cardiomyopathy being reversible, at least in some patients, with abstinence of alcohol, returning cardiac function towards baseline levels comparable with those of non-alcoholic cirrhotics. ${ }^{8}$

Myocardial diastolic dysfunction in cirrhosis can be exacerbated by simple manoeuvres such as a high sodium diet. ${ }^{2}$ More strenuous stresses may have more deleterious effects. Thus there have been occasional cases of unexpected death due to heart failure reported in cirrhotic patients undergoing treatment in whom cardiac disease had been excluded by routine investigations. These included post liver transplantation, ${ }^{45}$ transjugular intrahepatic portosystemic stent shunt, ${ }^{46}$ and surgical portocaval shunts. ${ }^{47}$ Therefore, it may be prudent to perform careful cardiological assessment in cirrhotic patients prior to any procedure that could potentially stress the heart.

In summary, patients with cirrhosis have a decreased cardiac response to exercise with reduced anaerobic capacity. The decreased cardiac performance is related, among other factors, to left ventricular hypertrophy and diastolic dysfunction. It is accompanied by a reduced chronotropic response, decreased stroke volume, and submaximal heart rate increase. The fact that subtle cardiac changes were detected in asymptomatic pre-ascitic and ascitic cirrhotic patients would suggest that cirrhotic cardiomyopathy can occur independent of the severity of liver dysfunction or haemodynamic changes. Therefore, this entity deserves further investigation.

The study was partly supported by grants from the Heart and Stroke Foundation of Ontario, Canada, grant No B4097.

1 Kowalski HJ, Abelmann WH. Cardiac output in Laennec cirrhosis. F Clin Invest 1953;32:1356-8.

2 Wong F, Liu P, Lilly L, et al. The role of cardiac structural and functional abnormalities in the pathogenesis of hyperdynamic circulation and renal sodium retention in cirrhosis. Clin Sci 1999;97:259-67.

3 Pozzi M, Carugo S, Boari G, et al. Functional and structural cardiac abnormalities in cirrhotic patients with and without ascites. Hepatology 1997;26:1131-7.

4 Finucci G, Desideri A, Sacerdoti D, et al. Left ventricular Finucci G, Desideri A, Sacerdoti D, et al. Left ventricular
diastolic dysfunction in liver cirrhosis. Scand $\mathcal{f}$ Gastroenterol diastolic dysfunction

5 Bernardi M, Rubboli A, Trevisani F, et al. Reduced cardiovascular responsiveness to exercise-induced sympathoadrenergic stimulation in patients with cirrhosis. $\mathcal{F}$ Hepatol 1991;12:207-16.

6 Laffi G, Barletta G, La Villa G, et al. Altered cardiovascular responsiveness to active tilting in nonalcoholic cirrhosis. Gastroenterology 1997;113:891-8.

7 Grose RD, Nolan J, Dillon JF, et al. Exercise-induced left ventricular dysfunction in alcoholic and non-alcoholic cirrhosis. F Hepatology 1995;22:326-32.

8 Guillo P, Mansourati J, Maheu B, et al. Long-term prognosis in patients with alcoholic cardiomyopathy and severe his in patients with alcoholic cardiomyopathy and severe heart failur 1276 -8.

9 Jones NL. Clinical exercise testing, 3rd edn. Philadelphia: WB Saunders, 1988.
10 Wong F, Liu P, Allidina Y, et al. Pattern of sodium handling and its consequences in pre-ascitic cirrhosis. Gastroenterology 1995;108:1820-7.

11 Otto CM, Pearlman AS. Echocardiographic evaluation of left and right systolic and diastolic function. In: Otto CM, Pearlman AS, eds. Textbook of clinical echocardiography. Philadelphia: WB Saunders, 1995:85-136.

12 Links JM, Becker LC, Shindledecker JG, et al. Measurement of absolute left ventricular volume from gated blood pool studies. Circulation 1982;65:82-90.

13 Wong F, Liu P, Tobe S, et al. Central blood volume in cirrhosis: measurement by radionuclide angiography. Hepatology 1994;19:312-21.

14 Goodman JM, McLaughlin PR, Plyley MJ, et al. Impaired cardiopulmonary responses to exercise in moderate hypertension. Can f Cardiol 1992;8:363-71.

15 Wasserman K. Diagnosing cardiovascular and lung pathophysiology from exercise gas exchange. Chest 1997;112: 1091-101.

16 Campillo B, Fouet P, Bonnet JC, et al. Submaximal oxygen consumption in liver cirrhosis. Evidence of severe functional aerobic impairment. I Hepatology 1990;10: 163-7.

17 Ritland S, Petlund CF, Knudsen T, et al. Improvement of physical capacity after long-term training in patients with chronic active hepatitis. Scand $\mathcal{f}$ Gastroenterol 1983;18: chronic $1083-7$.

18 Wasserman K, Hansen JE, Sue DY, et al. Principles of exercise testing and interpretation, 2nd edn. Philadelphia: Lea and Febiger, 1994

19 Lunseth JH, Olmstead EG, Forks G, et al. A study of heart disease in one hundred and eight hospitalized patients dying with portal cirrhosis. Arch Intern Med 1958;102:40513.

20 Hall EM, Olsen AY, Davis EE. Portal cirrhosis: clinical and pathological review of 782 cases from 16,600 necropsies. Am F Pathol 1953;29:993-1027.

21 Yamazaki T, Komuro I, Shiojima I, et al. The reninangiotensin system and cardiac hypertrophy. Heart 1996; 76(suppl 3):33-5.

22 Gray MO, Long CS, Kalinyak JE, et al. Angiotensin II stimulates cardiac myocyte hypertrophy via paracrine release of TGF-beta 1 and endothelin-1 from fibroblasts. Cardiovasc Res 1998;40:352-63.

23 Yamazaki T, Komuro I, Kudoh S, et al. Endothelin-1 is involved in mechanical stress-induced cardiomyocyte hypertrophy. F Biol Chem 1996;271:3221-8.

24 Kaye DM, Kelly RA, Smith TW. Cytokines and cardiac hypertrophy: roles of angiotensin II and basic fibroblast growth factor. Clin Exp Pharmacol Physiol Suppl 1996;3: S136-41.

25 Lindpainter K, Lund DD, Schmid PG. Effects of chronic progressive myocardial hypertrophy on indices of cardiac autonomic innervation. Circ Res 1987;61:55-62.

26 Kadambi VJ, Ponniah S, Harrer JM, et al. Cardiac specific over-expression of phospholamban alters calcium kinetics and resultant cardiomyocyte mechanics in transgenic mice. f Clin Invest 1996;97:533-9

27 Weber KT, Sun Y, Tyagi SC, et al. Collagen network of the myocardium: function, structural remodeling and regulatory mechanisms. F Mol Cell Cardiol 1994;26:279-92.

28 Lee SS, Marty J, Mantz J, et al. Desensitization of myocardial $\beta$-adrenergic receptors in cirrhotic rats. Hepatology 1990;12:481-5.

29 Ma Z, Meddings J, Lee SS. Membrane physical properties determine cardiac $\beta$-adrenergic receptor function in cirrhotic rats. Am 7 Physiol 1994;267:G87-93.

$30 \mathrm{Ma} \mathrm{Z}$, Lee SS, Meddings JB. Effects of altered cardiac membrane fluidity on $\beta$-adrenergic receptor signaling in rats with cirrhotic cardiomyopathy. 7 Hepatol 1997;26:90412 .

$31 \mathrm{Ma} \mathrm{Z}$, Miyamoto A, Lee SS. Role of the altered $\beta$-adrenoreceptor signal transduction in the pathogenesis of cirrhotic cardiomyopathy in rats. Gastroenterology 1996; 110:1191-8.

32 Saltin B. Hemodynamic adaptations to exercise. Am 7 Cardiol 1985;55:42-7D.

33 Naeije R, Melot C, Hallemans R, et al. Pulmonary hemodynamics in liver cirrhosis. Semin Respir Med 1985;7:164-70.

34 Castaing Y, Manier G. Hemodynamic disturbances and VA/Q matching in hypoxemic cirrhotic patients. Chest 1989;96:1064-9.

35 Agusti AG, Roca J, Rodriguez-Roisin R. Mechanisms of gas exchange impairment in patients with liver cirrhosis. Clin Chest Med 1996;17:49-66.

36 Agusti AGN, Roca J, Rodriguez-Roisin R, et al. Pulmonary hemodynamics and gas exchange during exercise in liver cirrhosis. Am Rev Respir Dis 1989;139:485-91.

37 Green DJ, O'Driscoll G, Blanksby BA, et al. Control of skeletal muscle blood flow during dynamic exercise: contribution of endothelium-derived nitric oxide. Sports Med 1996; 21:119-46.

38 Hickner RC, Fisher JS, Ehsani AA, et al. Role of nitric oxide in skeletal muscle blood flow at rest and during dynamic exercise in humans. Am f Physiol 1997;273:H405-10.

39 Bomzon A, Blendis LM. The nitric oxide hypothesis and the hyperdynamic circulation in cirrhosis. Hepatology 1994;20: $1343-50$.

40 Fredstrom SB, Khoruts A, Ayantuga O, et al. Nitric oxide production is not changed by transjugular intrahepatic production is not changed by transjugut
shunts. Gastroenterology 1999;116:A1211.

41 Balligand JL. Regulation of cardiac beta-adrenergic response by nitric oxide. Cardiovasc Res 1999;43:607-20. 
42 Paulus WJ, Shah AM. NO and cardiac diastolic function. Cardiovasc Res 1999;43:595-606.

43 Wong F, Logan AG, Blendis LM. Systemic hemodynamic, forearm vascular, renal and humoral response to sustained cardiopulmonary baroreceptor deactivation in cirrhosis. Hepatology 1995;21:717-24.

44 Urbano-Marquez A, Estruch R, Navarro-Lopez F, et al. The effects of alcoholism on skeletal and cardiac muscle. $N \mathrm{Engl}$ f Med 1989;320:409-15.
45 Rayes N, Bechstein WO, Keck H, et al. Causes of death after liver transplantation: an analysis of 41 cases in 382 patients. Zentralbl Chir 1995;120:435-8.

46 Lebrec D, Giuily N, Hadenque A, et al. Transjugular intrahepatic portosystemic shunt: comparison with paracentesis in patients with cirrhosis and refractory ascites: a randomized trial. F Hepatology 1996:25:135-44.

47 Franco D, Vons C, Traynor O, et al. Should portocaval shunt be reconsidered in the treatment of intractable ascites in cirrhosis? Arch Surg 1988:123:987-91. 